\title{
HISTORIA NATURAL DE LA TUBERCULOSIS. FISIOPATOGENIA DE LA INFECCIÓN Y LA ENFERMEDAD EN EL NIÑO
}

\author{
Natural history of tuberculosis. Pathogenesis of infection and disease in children
}

\author{
Dra. Magnolia Arango L \\ Neumóloga Pediatra \\ Profesora Titular, Universidad Nacional de Colombia
}

\begin{abstract}
NATURAL HISTORY OF TUBERCULOSIS. PATHOGENESIS OF INFECTION AND DISEASE IN CHILDREN
The multiple manifestations of tuberculosis (TB) are a result of the relationships between Mycobacterium tuberculosis, the host and the environmental, social and cultural conditions. In TB pathogenesis, three factors must be considered: the virulence of the bacillus, the potential of cellular destruction and caseous necrosis, and the immune response and hypersensitivity to the infection.

Key words: tuberculosis, mycobacterium tuberculosis, children, pathogenesis.
\end{abstract}

\section{RESUMEN}

La expresión múltiple de la tuberculosis se debe a la relación entre el Mycobacterium tuberculosis, el huésped y las condiciones medioambientales, sociales y culturales. En la patogenia de la tuberculosis se deben tener en cuenta tres factores: la virulencia del bacilo, la capacidad de producir destrucción celular y necrosis caseosa y la respuesta inmunitaria e hipersensibilidad a la infección.

Palabras clave: Tuberculosis, Mycobacterium tuberculosis, niños, patogenia.

\section{INTRODUCCIÓN}

La existencia de una infección o enfermedad como la tuberculosis (TB) infantil, resulta de una compleja interacción entre el huésped y sus características genéticas, inmunitarias, de nutrición y de salud, factores socioeconómicos, culturales y medio-ambientales en los que están inmersos el niño y su familia y la exposición a un bacilo como el Mycobacterium tuberculosis que ha ocasionado la enfermedad más antiguamente conocida.

Su historia natural comprende la secuencia biológica entre la exposición al bacilo, la infección, el paso a enfermedad y los desenlaces posibles mediados por diferentes condicionantes.

\section{MYCOBACTERIUM TUBERCULOSIS}

El género Mycobacterium apareció hace 150 millones de años hasta hacerse contemporáneo a los homínidos (3 millones de años); ha acompañado a la especie humana desde que inició su asentamiento durante el Neolítico en África. El actual complejo Mycobacterium tuberculosis (M. tuberculosis, M. bovis, M. africanum, M. microti, M. canetti), existe desde hace aproximadamente 15.000 - 20.000 años, con evolución

\section{Correspondencia:}

Dra Magnolia Arango L

Ciudad Universitaria, Facultad de Medicina

Universidad Nacional de Colombia

E-mail:marangode@unal.edu.co,magnoliarango@gmail.com paralela a la del Homo sapiens. Su evidencia paleontológica se ha encontrado en restos vertebrales humanos de hace $7.000 \mathrm{a}$ 11.000 años. La progresión y transmisión como patología deriva de la urbanización de las comunidades humanas.

Se ha encontrado en restos humanos americanos prehispánicos (Canadá, Estados Unidos, México, República Dominicana, Panamá, Colombia, Venezuela, Perú, Chile y Argentina) por estudios patológicos y visualización de bacilos. Con la detección del ADN bacilar se ha confirmado en momias indígenas precolombinas (Chile, Perú y Colombia) (1). Las formas graves frecuentes en poblaciones indígenas americanas se atribuyen a débil inmunidad previa, a Mycobacterium libres o poco virulentos de $\mathrm{M}$. tuberculosis, o M. bovis que explicarían la susceptibilidad de indígenas modernos a bacilos virulentos 0 cepas europeas.

En la familia Mycobacteriacea hay tres complejos: el Mycobacterium tuberculosis (Bacilo de Koch, BK), el Mycobacterium leprae y el Mycobacterium no tuberculosis. Del complejo Mycobacterium tuberculosis, produce enfermedad en la mayoría de casos la variedad humana, el M. tuberculosis; el $\mathrm{M}$. bovis ocasionalmente produce patología por ingestión de leche contaminada.

De los Mycobacterium no tuberculosis (M. avium, M. intracellulare, siendo más de 100 especies), muchos son saprófitos, otros oportunistas y algunos patógenos (2). Generalmente no son virulentos en huéspedes inmunocompetentes; producen Mycobacteriosis en inmunodeficientes o con enfermedades de base.

El M. tuberculosis es la bacteria más letal que ha 
existido en la historia a pesar de ser inmóvil, de crecimiento lento, sin vectores 0 reservorios animales muy eficientes, carecer de esporas, endotoxinas o enzimas histolíticas; además es dependiente del aire para transmitirse (sobrevive pocas horas al sol y la ventilación). La potencialidad de lesionar se debe a tres factores: capacidad de enfermar a través de toda la vida del huésped, habilidad de producir enfermedad pulmonar y diseminarse por tos y el hecho de no inmovilizar al huésped ni producirle necesariamente la muerte. A su patogenicidad también contribuyen la alta densidad poblacional humana, las precarias condiciones de vida y el debilitamiento del huésped (3).

El M. tuberculosis infecta sin ser dominado fácilmente por los mecanismos de defensa debido a su pared lipídica (ácidos micólicos) que lo hace resistente a antibióticos usuales y le confieren las propiedades de tinción (retienen coloración roja al tratarlos con ácido-alcohol, técnica de Ziehl Nielsen). Es resistente al frío, la desecación y la congelación y sensible a la luz solar directa, la ventilación y el calor húmedo y es destruido por la pasteurización $\left(>80^{\circ} \mathrm{C}\right)$. Es aerobio estricto, se multiplica cada 20 horas en lesiones con suficiente 02 y $\mathrm{pH}$ de 7,4 (por ejemplo en cavernas) pero puede sobrevivir en ambientes anaerobios en donde queda en estado durmiente con escasa actividad durante años o décadas; esta adaptabilidad al medio externo mantiene la endemia pues los sanos infectados constituyen un reservorio; la multiplicación lenta del bacilo origina manifestaciones inespecíficas que originan consultas tardías, perpetuando la transmisión (4).

El Mycobacterium tuberculosis puede afectar varias especies animales pero en el humano es endémico y se perpetúa como patógeno.

La patogenicidad y capacidad de infectar y enfermar del BK, se relacionan con su virulencia y habilidad de eludir la destrucción por los macrófagos no sensibilizados al iniciar la infección así como a la inducción de hipersensibilidad celular retardada.

\section{MEDIO AMBIENTE Y FACTORES DE RIESGO SOCIALES, ECONÓMICOS Y CULTURALES}

La TB ocasiona aún gran morbilidad y mortalidad; en muchas regiones del mundo es endémica, en algunas epidémica; afecta a los más pobres en países de bajos recursos y a quienes están en condiciones de pobreza en paises desarrollados (5). Afecta directamente a los niños y sus familias haciéndose más vulnerables cuanto más haya precariedad socio-económica y de sus condiciones de vida (vivienda, hacinamiento, alimentación); incrementan el riesgo el desplazamiento por conflictos bélicos y la explotación infantil.

La ausencia y baja cobertura de inmunización con BCG, la coinfección TB/VIH, la resistencia a medicamentos, la inmunosupresión y enfermedades de base, han aumentado la susceptibilidad de los niños en quienes la TB es reflejo de la prevalencia en adultos y evento centinela que indica transmisión reciente y progresiva del BK.

Según OMS/OPS, los mayores factores de riesgo en niños para infectarse, enfermar o tener una progresión grave, son:
- Contacto intradomiciliario frecuente con bacilíferos

- Edad inferior a cinco años

- Coinfección con VIH

- Desnutrición grave

Hay poblaciones en mayores condiciones de vulnerabilidad: los indígenas y afroamericanos marginados e igualmente los desplazados y los habitantes de calle (6). Aunque todos estos factores son trascendentes, no se puede olvidar que todos los niños son susceptibles pues no tienen inmunidad innata.

El desconocimiento de la situación real de la enfermedad y sus riesgos, la insuficiente detección de sintomáticos respiratorios, infectados y enfermos y los tratamientos inadecuados, contribuyen al aumento de la exposición al bacilo y a que no se corte la cadena de transmisión. En varios países de Latinoamérica hay niños no registrados oficialmente ni cobijados por ningún sistema de salud y no se benefician de actividades de saneamiento, educación, prevención o vacunación. En TB, Ios condicionantes y la enfermedad actúan en doble sentido: sus determinantes hacen perder factores protectores y empeoran los de riesgo produciéndose la patología; a su vez, ésta desencadena eventos que deterioran más el estado precario de salud y de forma de vida. Alguien carente de recursos, desnutrido y enfermo, va a agravar todos los componentes de la enfermedad misma: deterioro de las condiciones de vida material, desempleo, desnutrición, etc. y se crea un nefasto círculo vicioso. Se suma el estigma social que aún pesa sobre los enfermos quienes por miedo al rechazo, la discriminación y el marginamiento, consultan tardíamente, permanecen sin diagnóstico o no son beneficiarios de ninguna actividad de apoyo (7).

Siempre se ha considerado que la TB es una "enfermedad de la pobreza" y aunque es una entidad infectocontagiosa, se reconoce planamente que las condiciones socioeconómicas, culturales y ambientales y la exclusión social en las que viven las poblaciones vulnerables, favorecen su ocurrencia (8). En regiones donde hay menos recursos y poco acceso a la atención hay mayor número de casos, más patologías que aplazan el diagnóstico, dificultades para comprobarlo y se subestima la carga de la enfermedad; no conocemos con precisión el número de casos reales en regiones donde el problema es mayor (9). Por ser el estado de salud y bienestar de los niños dependiente de la familia y en especial de las madres, conviene recordar que en muchos paises su salud es bastante precaria; se estima que en el mundo hay 2.900.000 casos de TB en mujeres, con mortalidad de 410.000 (10), hecho que conlleva consecuencias graves: orfandad, pobreza, abandono, etc (11).

\section{HUÉSPED}

La genética del bacilo define la capacidad de infección o enfermedad; las características genéticas del huésped también determinan la eficiencia de movilización de las defensas y de respuesta adecuada. El papel del genotipo y fenotipo para infectarse 0 enfermar o no con el BK está en estudio. Habría genes programados para regular la resistencia natural, la actividad macrofágica, la presentación de antígenos, la producción de ácido nítrico, de vitamina D como inmunoregulador, la modulación 
del interferón y las citocinas; estos procesos están relacionados además con el complejo mayor de histocompatibilidad (HLA) y determinarían la susceptibilidad diferencial y heterogeneidad de la TB. Se están estudiando factores epigenéticos medioambientales relacionados (12).

Los niños están más expuestos tempranamente en el hogar (cada enfermo bacilífero contagia entre 16 a 20 personas de su entorno) y son más vulnerables a la infección; su respuesta inmune aún en sanos es insuficiente para eliminar todos los BK de la infección, no son eficientes en contenerlos en el granuloma inicial y pueden desarrollar enfermedad severa y diseminada.

Los factores de riesgo de infección o de progresión a enfermedad se relacionan directamente con el sistema inmune del niño quién puede infectarse en cualquier edad pero la probabilidad, frecuencia y gravedad son mayores cuanto menor es. El $50 \%$ de los expuestos se infectan, con mayor riesgo de progresión a enfermedad grave, extrapulmonar o diseminación en $<2$ años, desnutridos, adolescentes e inmunodeficientes (13). La mortalidad en $<1$ año es del $50 \%$. La mitad de enfermos que no reciban tratamiento morirán en los próximos 5 a 8 años.

Conviene enfatizar que aumentan la susceptibilidad, progresión, gravedad y mortalidad: la desnutrición, la deficiencia de vitamina D; la ausencia de BCG; la infección por VIH (20 veces más), los inmunosupresores, antagonistas TNF (Factor de Necrosis Tumoral) y las condiciones debilitantes: diabetes, falla renal o hepática, cáncer, neumoconiosis y las infecciones virales (sarampión, varicela, influenza) y bacterianas (tos ferina).

Hay tres desenlaces posibles del contacto: el niño expuesto no infectado, la infección latente y quién progresa a enfermedad activa. Es importante definir estos estadíos; la dificultad radica en que la transición entre infección latente y enfermedad es un continum que a veces es indefinido y subclínico.

\section{FISIOPATOGENIA}

Transmisión, contagio, inoculación, infección y enfermedad

La principal vía de transmisión y contagio es por aerosoles que contienen bacilos viables, inhalados por personas susceptibles en general en espacios cerrados. En aire ambiente abierto los bacilos se dispersan y mueren por la luz ultravioleta; algunos permanecen viables en partículas aerosolizadas de nuevo. La fuente de infección casi siempre es un adulto 0 adolescente con lesiones pulmonares comunicadas al aire que expelen partículas infectantes formando aerosoles transportadores del BK a través de la tos, el habla, la risa, el canto, los estornudos y la expectoración.

La contagiosidad depende de la severidad del cuadro, la frecuencia de la tos, el tamaño del inóculo y la cepa y virulencia del $\mathrm{M}$. tuberculosis y si la persona contagiosa recibe 0 no tratamiento anti-TB.

\section{INFECCIÓN}

Los niños se infectan casi siempre por exposición doméstica o contacto estrecho con un bacilífero del medio familiar, generalmente padres o convivientes enfermos; otras fuentes son: cuidadores externos, maestros, expendedores de comida, etc. Los bacilíferos son la mayor fuente de transmisión y contagio.

Otras vías de infección de menor frecuencia son la ingestión, en especial de M. bovis o por contaminación accidental por las diferentes micobacterias.

La inmunidad natural innata contra la infección consiste en mecanismos como la nutrición normal, la integridad cutánea y de mucosas respiratorias y digestivas, sus secreciones normales, la anatomía de las vías respiratorias, el clearance mucociliar, la flora residente y las respuestas inflamatorias e inmunológicas iniciales, representadas por macrófagos, linfocitos (NK, natural killers) y neutrófilos.

Las microgotas inhaladas que contienen 2-3 bacilos, alcanzan los alvéolos. En niños inmunocompetentes la respuesta se inicia con macrófagos alveolares no activados y células dendríticas; los antígenos bacilares alteran las células epiteliales y producen una inflamación local y reclutamiento celular (formación de foco de consolidación neumónica); las opsoninas inducen la adherencia de los bacilos a receptores específicos del macrófago que se activa para fagocitarlos; por razones desconocidas, ya englobados por los fagosomas, no son lisados como otras bacterias, sino que inician su crecimiento y reproducción; los macrófagos cargados con bacilos migran por linfáticos hacia ganglios vecinos; allí presentan los antígenos bacilares a los linfocitos T CD4 y CD8 nativos (indispensables para contener la infección) y activan los linfocitos $T$ que se dividen en TH1 (liberan citoquinas, interleuquinas, TNF-b e IFNe interferon gama) y TH2 (IL-1, IL-4, IL-5 y otras que estimulan los linfocitos $\mathrm{B}$ a fin de controlar la multiplicación y diseminación bacilar.

Cuando se pierden estas funciones, los bacilos intracelulares siguen multiplicándose hasta necrozar la célula huésped y liberarse hacia el alvéolo; las células de memoria migran hacia el sitio de la infección y median una respuesta de monocitos y macrófagos para matar a los bacilos posiblemente por liberación local de óxido nítrico; los bacilos liberados pueden alcanzar ganglios linfáticos u otros sitios a través de la vía hematógena.

Al segundo día del ingreso bacilar se inicia la formación del granuloma que crece en 2 semanas a $1 \mathrm{~mm}$ (visible como un punto grisáceo) y se originan células gigantes multinucleadas centrales o de Langerhans, que contienen a menudo bacilos en su citoplasma, rodeadas por macrófagos distorsionados llamados epiteloides y linfocitos y fibroblastos que forman un tejido fibroso circunvalar encapsulando la lesión. Posiblemente por anoxia central y productos del bacilo mismo, en varios días puede ocurrir necrosis caseosa y al morir los macrófagos, liberar nuevos micro-organismos; la mayoría de veces se contiene la lesión y desaparece o deja una cicatriz imperceptible o una calcificación que atrapa los bacilos y puede ser visualizada radiológicamente a los 6 meses.

Este período de infección (primoinfección TB) suele ser asintomático, no transmisible y deja una huella inmunológica de hipersensibilidad detectable por la prueba de tuberculina (PPD). El periodo de latencia o incubación entre la llegada del bacilo y la evidencia de la infección es 8 a 10 semanas. El foco parenquimatoso, el compromiso línfático y del ganglio satélite, constituyen el Complejo primario de Ghon que puede dejar una 
cicatriz frecuentemente calcificada, visible radiológicamente (Complejo de Ranke). Cuando hay una adecuada respuesta inmune protectora a la infección primaria, se desarrolla un foco fibroso y en niños mayores un foco de caseificación y posterior fibrosis; los ganglios regionales cicatrizan en meses; la linfadenopatía puede persistir por años y en ella existir algunos bacilos viables que eventualmente producirán enfermedad si la capacidad inmune declina.

La formación de granulomas o caseum y la calcificación son útiles para contener la infección y prevenir la diseminación (el medio extracelular ácido, carente de 02, impide la multiplicación bacilar y salida del foco inicial); si la lesión avanza, la mayoría de veces lentamente, va alterando el órgano y los síntomas aparecen cuando el daño es importante; en niños y jóvenes la potencialidad de reparación del tejido pulmonar puede minimizar la alteración y dejar escasa huella si se administra tratamiento adecuado.

Los niños presentan formas de primoinfeción en la mayoría de casos y su curación sucede en el 95\% de los inmunocompetentes infectados. Las formas secundarias de reactivación o reinfección son frecuentes en adultos.

La transmisión de Mycobacterium no tuberculosis al humano proviene de fuentes ambientales como aerosoles provenientes de agua estancada, partículas de tierra 0 polvo. Los animales parecen tener un rol importante en la transmisión a humanos (14).

\section{LATENCIA}

El organismo infectado moviliza sus mecanismos de defensa para contener los bacilos pero algunos pueden sobrevivir y quedar en un estado de quiescencia, durmientes 0 en "hibernación" dentro del macrófago el cual al no detectar crecimiento bacteriano, deja de enviar estímulos a los CD8 y $\mathrm{CD} 4$, haciendo que el bacilo se haga invisible para el sistema inmune y pueda reactivarse después; en este estado de latencia, aunque no es inerte, es imposible verlo o cultivarlo; su ADN si es detectable. Esta situación se denomina infección latente, en la cual el niño está asintomático pero la infección bacilar puede demostrarse por la positividad tuberculínica u otras pruebas. Por ser de especial relevancia en los niños debido a la posibilidad de pasar en forma inadvertida hacia una enfermedad pulmonar 0 diseminada, debe ser cuidadosamente controlada.

La respuesta inmunológica y el riesgo de infectarse con el M. tuberculosis son complejos; parece existir cierta resistencia natural pues el $50 \%$ de los expuestos no se infectan pero la base inmunogenética de esta susceptibilidad diferencial, asi como la infección latente es poco clara (15).

\section{ENFERMEDAD}

Cuando confluyen factores de riesgo, alrededor del $5 \%$ de los niños infectados se enferman apareciendo síntomas respiratorios, constitucionales o generales, el $90 \%$ de casos en el primer año después de la infección; entre el 5\%-10\% desarrolla la enfermedad posteriormente.

Si la contención de la infección falla, el complejo primario puede evolucionar hacia la enfermedad; en la mayoría de casos el compromiso es parenquimatoso pulmonar; el material necrótico se licúa y convierte en una secreción viscosa con bacilos; el granuloma en crecimiento destruye el tejido vecino y erosiona vasos y paredes alveolares y el material bacilar pasa a otros alveolos con diseminación pulmonar primaria (neumonía TB) o reblandecimiento de un ganglio drenado hacia un bronquio (diseminación bronquial primaria); el material con bacilos puede llegar a linfáticos o vasos y sembrarse prácticamente en cualquier tejido u órgano por lo cual los niños pueden presentar TB pulmonar progresiva o formas miliares y extrapulmonares (ganglionar, meníngea, pleural, pericárdica, abdominal, osteoarticular, cutánea, renal).

Los niños esporádicamente presentan cavernas o formas "abiertas" de TB, bacilíferas (especialmente los adolescentes); en general el compromiso pulmonar infantil es de consolidación parenquimatosa o complejo primario evolutivo; las poblaciones bacilares son de escaso número o paucibacilares por lo cual se consideran en general poco bacilíferos.

Hay un aumento preocupante de formas congénitas transmitidas vía transplacentaria hematógena, por ingestión de líquido amniótico infectado o secreciones durante el parto y de la neonatales (exposición del recién nacido a bacilífero, usualmente la madre) (16). La leche materna no transmite el Mycobacterium tuberculosis.

\section{CONCLUSIÓN}

La comprensión de la historia natural y la fisiopatogenia de la infección y la enfermedad infantil por TB permiten comprender su amplio espectro clínico y es la manera posible de abordar su diagnóstico (17) pues la entrada al organismo, la complejidad de la respuesta inmunológica que desencadena y la forma de compromiso de tejidos y órganos en un espectro tan amplio, requieren del conocimiento claro de su existencia, formas heterogéneas de presentación y del correcto diagnóstico; de ello somos responsables los médicos, en especial quienes atendemos niños.

\section{El autor declara no presentar conflicto de intereses}




\section{REFERENCIAS}

1. Sánchez Luna M, Moreno Hernando J, Botet Mussons F, Fernández Lorenzo JR, Herranz Carrillo G, Rite Gracia S, Salguero García E, Echaniz Urcelay I. Displasia broncopulmonar: definiciones y clasificación. An Pediatr 2013; 79:262

2. Palominos MA, Morgues M, Martinez F. Management of infants with Chronic Lung Disease of prematurity in Chile. Early Hum Dev 200; 81: 151-4

3. Bancalari A. Actualización en Presentación y Patogénesis de la Displasia Broncopulmonar. Rev Chil Pediatr 2009; 80 : 213-224

4. Jobe AH. The new bronchopulmonary dysplasia. Curr Opin Pediatr 2011;23:167-72

5. Northway WH Jr, Rosan RC, Porter DY. Pulmonary disease following respiratory therapy of hyaline membrane disease: bronchopulmonary dysplasia. N Engl J Med 1967; 276: 357-68

6. Zeitlin P, Nogee L. Etiology of Bronchopulmonary Dysplasia: Before Birth. Pediatr Allergy Immunol Pulm 2011; 24: 2125

7. Gien J, Kinsella J. Pathogenesis and treatment of bronchopulmonary dysplasia. Curr Opinion Pediatr 2011; 23:305-313

8. Mourani P, Abman S. Pulmonary vascular disease in bronchopulmonary dysplasia.: pulmonary hypertension and beyond. Curr Opin Pediatr 2013;25: 329-337

9. Baraldi E, Filippone M. Chronic Lung Disease after Premature Birth. N Engl J Med 2007; 357:1946-55

10. American Thoracic Society Documents Statement on the Care of the Child with Chronic Lung Disease of Infancy and Childhood. Am J Respir Crit Care Med; 2003: 356-396

11. Jobe A, Bancalari E. NICHD/NHLBI/ORD Workshop Summary. Bronchopulmonary Dysplasia. Am J Respir Care Med 2001; 163: 1723- 1729

12. Bancalari E, Claure N. Definitions and diagnostic criteria for bronchopulmonary dysplasia. Semin Perinatol, 2006, 30: 164-170

13. Fernández $C$, Fajardo $C$, Favareto $M$, Hoyos A, Jijón-Letort $F$, Carrera M, Yllescas M, Romero M. Oxygen dependency as equivalent to bronchopulmonary dysplasia at different altitudes in newborns $\leq 1500 \mathrm{~g}$ at birth from the SIBEN network Members of Iberoamerican Society of Neonatology network. J Perinatol. 2014; 34:538-42

14. 14. MINISTERIO DE SALUD. Guía Clínica Displasia Broncopulmonar del Prematuro. Santiago: MINSAL, 2009 . Web.minsal.cl/portal/url/ item/721fc45c974a9016e04001011f0113bf.pdf

15. Ola Didrik Saugstad, Dagfinn Aune. Optimal Oxygenation of Extremely Low Birth Weight Infants: A Meta-Analysis and Systematic Review of the Oxygen Saturation Target Studies. Neonatol 2014;105:55-63

16. Poets CF. When do infants need additional inspired oxygen? A review of the current literature. Pediatr Pulmonol. 1998; 26:424-8

17. Kotecha S, Allen J. Oxygen therapy for infants with chronic lung disease. Arch Dis Child Fetal Neonatal 2002; 87:F11-4

18. 18. Primhak RA. Discharge and aftercare in chronic lung disease of the newborn. Semin Neonatol 2003;8:117-26 